$U D C 519.6$

\title{
A DOUBLE-LAYER POTENTIAL APPROACH FOR PLANAR CAUCHY PROBLEMS FOR THE LAPLACE EQUATION
}

\author{
R. Chapko ${ }^{1}$, B. T. Johansson ${ }^{2}$, M. Shtoyko ${ }^{1}$ \\ ${ }^{1}$ Ivan Franko National University of Lviv, \\ Universytetska str., 1, Lviv, 79000, Ukraine, e-mail: roman.chapko@lnu.edu.ua \\ ${ }^{2}$ Mathematics, ITN, Campus Norrköping, Linköping University, \\ 601 74, Norrköping, Sweden, e-mail: tomas.johansson@liu.se
}

\begin{abstract}
We consider the Cauchy boundary value problem for the Laplace equation in a planar bounded doubly-connected domain. Our goal is to reconstruct the Cauchy data on the interior boundary from given Cauchy data on the outer boundary. It is an example of the linear ill-posed inverse problem. Using the indirect integral equation method based on a double-layer representation for the Cauchy problem we receive a system of integral equations to be solved for two unknown densities. It is shown that the system has a unique solution for a dense set of data. Next we parametrize this system to periodical integral equations and separate the given hypersingularity in some kernels as a special weight function. The numerical solution of integral equations is realized by Nystroem method based on trigonometrical quadrature rules. As result the full discrete system of linear equations with respect to approximation values of unknown densities in the quadrature points is received. Since this system is ill-posed it is solved by Tikhonov regularization. The value of the regularization parameter is chosen by trial and error. It gives us the possibility to receive the stable solution. The influence of the discretization is also included in a brief error analysis. Results of numerical experiments for the reconstruction of the function and its normal derivative on the interior boundary show that accurate approximations can be obtained with the double-layer method also for the noisy Cauchy data. In the case of noisy data, random pointwise errors are added to the function values on the outer boundary with the percentage given in terms of the least squares norm.
\end{abstract}

Key words: Cauchy problem, double-layer potential, hypersingular integral equation, $\mathrm{Ny}-$ ström method, Tikhonov regularization.

\section{INTRODUCTION}

The Cauchy problem for the Laplace equation has been studied for over a century and serves as a typical example of a linear inverse ill-posed problem. The Cauchy problem has several important applications, for example, in cardiology, corrosion detection, electrostatics, geophysics, leak identification, non-destructive testing and plasma physics, for references see [2], where also references to some regularizing solution methods are given. We shall not go into details or list further references on history and properties of Cauchy problems, but only state that data are assumed compatible such that there exists a solution. We focus on recent layer potential methods for the Cauchy problem.

Layer potential based methods leading to first kind Fredholm integral equations have been successful for well-posed boundary value problems both for theoretical investigations and numerical calculations. For ill-posed Cauchy problems reduction via layer potentials is more recent and less studied. Starting with the work [2] and building on results from [1], a string of works have been produced showing that a single-layer approach can be applied also to ill-posed Cauchy problems, and is in fact simple and straightforward

(C) Chapko R., Johansson B.T., Shtoyko M., 2020 
but yet mathematically rigorous. This single-layer method has been implemented and tested in various planar domains both doubly and simply connected as well as unbounded, for details and references see the overview in [3]. The method is not restricted to the Laplace equation but can also be applied to, for example, the similar ill-posed problem in elasticity and for other stationary and non-stationary fields of various physical processes, see $[4,5]$.

To further complete the research on layer based methods for ill-posed Cauchy problems, it is natural to consider the use of a double-layer instead of a single-layer. Therefore, we present a double-layer method for the Cauchy problem for the Laplace equation in planar doubly-connected domains. As is known for the Dirichlet problem for the Laplace equation in multiply connected domains, the classical double-layer potential has to be adjusted with additional terms to generate a unique solution, see [13, Chapt. IV, Sect. 31] and [7]. The similar modification is adopted here. Using this layer representation leads to a hypersingularity appearing in the resulting system of integral equations. We examine the influence of this singularity and its effect on the regularization.

For the outline of this work, in Section 2, we present what is termed the indirect integral method based on a double-layer representation for the Cauchy problem, leading to a system of integral equations to be solved for two unknown densities. The operator corresponding to this system is injective and has dense range, see Theorem 1 . In Section 3 , we show how to discretize the obtained system using the Nyström method and discuss the stable solution of it via Tikhonov regularization. The influence of the discretization is also included in a brief error analysis. Section 4 is devoted to numerical investigations; numerical examples are given showing that accurate solutions can be obtained also in the case of noisy Cauchy data. Some conclusions are stated in Section 5 .

Before undertaking the outlined work, we end this section by formulating more precisely the Cauchy problem we study. Let $D_{2} \subset \mathbb{R}^{2}$ be a bounded domain with boundary curve $\Gamma_{2}$. This curve is assumed to be simple closed and sufficiently smooth; this means in particular that the curve has no self-intersections and is connected, and the curve itself has no boundary. Let then $\Gamma_{1}$ be a simple closed (smooth) curve lying wholly within $D_{2}$ with the interior of $\Gamma_{1}$ being denoted $D_{1}$. The solution domain $D$ is the region between the two curves $\Gamma_{1}$ and $\Gamma_{2}$, thus $D=D_{2} \backslash \bar{D}_{1}$. The outward unit normal to the boundary is denoted by $\nu$, see further Fig. 1 for an example of the configuration. We assume additionally that the origin does not belong to the domain $D$.

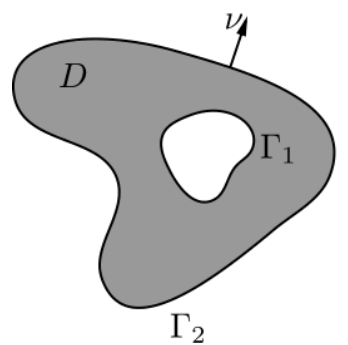

Fig. 1. Example of a solution domain $D$

Let $u \in C^{2}(D) \cap C^{1}(\bar{D})$ be harmonic, that is a solution to the Laplace equation

$$
\Delta u=0 \quad \text { in } D
$$


Chapko R., Johansson B. T., Shtoyko M.

with $u$ also satisfying the following two boundary conditions on the outer curve $\Gamma_{2}$,

$$
u=f \quad \text { on } \Gamma_{2} \quad \text { and } \quad \frac{\partial u}{\partial \nu}=g \quad \text { on } \Gamma_{2} .
$$

This is the Cauchy problem that we shall present a double-layer approach for to generate a stable numerical approximation of the solution $u$.

Before ending this section, we note that our suggested approach is different from the one given by Sun [14] for the Cauchy problem. In [14], an additional domain $B$ containing the given domain $D$ is introduced, and the solution is represented as a doublelayer potential over the boundary $\partial B$. As a result, ill-posed integral equations of the first kind are obtained. The similar idea when using ill-posed integral equations in potential theory are presented by Kress, see for example, [11, p. 343].

\section{ILL-POSED SYSTEM OF HYPERSINGULAR INTEGRAL EQUATIONS}

The use of a double-layer potential for the interior Dirichlet problem in multiply connected domains involves some modification of the representation of the solution. Modification is necessary to ensure the well-posedness of the obtained system of integral equations since there are non-trivial null solutions. A modification is suggested by Mikhlin in [13, Chapt. IV, Sect. 31] and successfully used by other authors for example $[7,9]$. Therefore, following [13], we seek the solution to the Cauchy problem (1)-(2) in the following form

$$
u(x)=\sum_{\ell=1}^{2} \int_{\Gamma_{\ell}} \phi_{\ell}(y) \frac{\partial \Phi(x, y)}{\partial \nu(y)} d s(y)+A \ln |x|^{2}, \quad x \in D
$$

where $\phi_{1} \in C\left(\Gamma_{1}\right)$ and $\phi_{2} \in C\left(\Gamma_{2}\right)$ are unknown densities (continuous densities imply a straightforward interpretation of the boundary integrals), $A \in \mathbb{R}$ is an unknown constant and

$$
\Phi(x, y)=\frac{1}{2 \pi} \ln \frac{1}{|x-y|}, \quad x \neq y
$$

is the fundamental solution of the Laplace equation in $\mathbb{R}^{2}$.

Using the classical jump properties of the double-layer potential and its normal derivative, the representation (3) satisfies (1)-(2) provided that the two densities $\phi_{1}$ and $\phi_{2}$ together with the constant $A$ solve the following system

$$
\left\{\begin{array}{l}
-\frac{1}{2} \phi_{2}(x)+\sum_{\ell=1}^{2} \int_{\Gamma_{\ell}} \phi_{\ell}(y) \frac{\partial \Phi(x, y)}{\partial \nu(y)} d s(y)+A \ln |x|^{2}=f(x), \quad x \in \Gamma_{2}, \\
\sum_{\ell=1}^{2} \frac{\partial}{\partial \nu(x)} \int_{\Gamma_{\ell}} \phi_{\ell}(y) \frac{\partial \Phi(x, y)}{\partial \nu(y)} d s(y)+2 A \frac{x \cdot \nu(x)}{|x|^{2}}=g(x), \quad x \in \Gamma_{2}, \\
\int_{\Gamma_{1}} \phi_{1}(y) d s(y)=0 .
\end{array}\right.
$$

We show below that the above system has at most one solution for a dense set of data. 
We introduce the following boundary integral operators

$$
\begin{gathered}
\left(T_{i j} \mu\right)(x)=\frac{\partial}{\partial \nu(x)} \int_{\Gamma_{j}} \mu(y) \frac{\partial \Phi(x, y)}{\partial \nu(y)} d s(y), x \in \Gamma_{i}, \\
\left(D_{i j} \mu\right)(x)=\int_{\Gamma_{j}} \mu(y) \frac{\partial \Phi(x, y)}{\partial \nu(y)} d s(y), x \in \Gamma_{i},
\end{gathered}
$$

and

$$
Q_{i} \phi=\int_{\Gamma_{i}} \phi(y) d s(y)
$$

together with the two functions

$$
G_{i}(x)=\ln |x|^{2}, \quad \tilde{G}_{i}(x)=2 \frac{x \cdot \nu(x)}{|x|^{2}} \quad x \in \Gamma_{i},
$$

where $i, j=1,2$.

Then we rewrite the system (5) in the following operator form

$$
\left\{\begin{array}{l}
D_{21} \phi_{1}+\left(-\frac{1}{2} I+D_{22}\right) \phi_{2}+A G_{2}=f \\
T_{21} \phi_{1}+T_{22} \phi_{2}+A \tilde{G}_{2}=g \\
Q_{1} \phi_{1}=0
\end{array}\right.
$$

The operator matrix of the left-hand side in (8) is denoted by $B$ and is considered as a mapping $B: L^{2}\left(\Gamma_{1}\right) \times L^{2}\left(\Gamma_{2}\right) \times \mathbb{R} \rightarrow L^{2}\left(\Gamma_{2}\right) \times L^{2}\left(\Gamma_{2}\right) \times \mathbb{R}$, where

$$
B=\left(\begin{array}{ccc}
D_{21} & -\frac{1}{2} I+D_{22} & G_{2} \\
T_{21} & T_{22} & \tilde{G}_{2} \\
Q_{1} & 0 & 0
\end{array}\right) .
$$

The system (8) corresponds to the ill-posed Cauchy problem (1)-(2), and will therefore inherit the ill-posedness. Thus, rather than showing well-posedness, it is important that the operator $B$ has properties such that Tikhonov regularization can be applied for the stable solution. We therefore show the following result.

Theorem 1. The operator $B$ defined in (9) is injective and has dense range in the space $L^{2}\left(\Gamma_{2}\right) \times L^{2}\left(\Gamma_{2}\right) \times\{0\}$.

Proof. Assume that $B \phi=0$, where $\phi=\left(\phi_{1}, \phi_{2}, A\right) \in L^{2}\left(\Gamma_{1}\right) \times L^{2}\left(\Gamma_{2}\right) \times \mathbb{R}$. The element $u$ given by the modified double-layer potential $(3)$ is then a solution to the Laplace equation in the interior of $D$ with $\left.u\right|_{\Gamma_{2}}=0$ and $\partial u /\left.\partial \nu\right|_{\Gamma_{2}}=0$. Thus, by Holmgren's theorem, $u=0$ in $\bar{D}$. Consider then the Dirichlet boundary value problem in the domain $D$ with homogeneous boundary conditions. We have a representation of the solution to that Dirichlet problem in the form of the modified double-layer potential (3) with the last condition of (8) being satisfied by the assumption $B \phi=0$. According to [13, Chapt. IV, Sect. 31], we can therefore conclude that the densities $\phi_{1}=0$ and $\phi_{2}=0$ as well as $A=0$ in the representation (3). Hence, the operator $B$ is injective.

We then show that $B$ has dense range in $L^{2}\left(\Gamma_{2}\right) \times L^{2}\left(\Gamma_{2}\right) \times\{0\}$. Let $f_{2}$ be a fixed smooth function on $\Gamma_{2}$ and $f_{1}$ an arbitrary smooth function on $\Gamma_{1}$. The solution $u$ to the 
Chapko R., Johansson B. T., Shtoyko M.

ISSN 2078-5097. Вісн. Львів. ун-ту. Сер. прикл. матем. та інф. 2020. Вип. 28

Dirichlet problem having $\Delta u=0$ in $D$ and $u=f_{2}$ on $\Gamma_{2}$ and $u=f_{1}$ on $\Gamma_{1}$, can, according to [13, Chapt. IV, Sect. 31], be represented as the modified double-layer potential (3) with the last condition in (8) satisfied.

Let $g_{2}=g_{2}\left(f_{1}\right)$ be the restriction to $\Gamma_{2}$ of the normal derivative of this modified double-layer potential. We claim that as $f_{1}$ varies arbitrarily, $g_{2}\left(f_{1}\right)$ forms a dense set in $L^{2}\left(\Gamma_{1}\right)$. Assume on the contrary that $g_{2}\left(f_{1}\right)$ does not form a dense set. Then there exists a non-trivial element $z_{2}$ with

$$
\int_{\Gamma_{2}} g_{2}\left(f_{1}\right)(y) z_{2}(y) d s(y)=0
$$

for every choice of $f_{1}$.

Let $v$ be a solution to the Dirichlet problem having $\Delta v=0$ in $D$ and $v=z_{2}$ on $\Gamma_{2}$ and $v=0$ on $\Gamma_{1}$. Applying Green's formula utilizing the boundary conditions for $u$ and $v$ together with (10) render

$$
\int_{\Gamma_{2}} f_{2}(y) \frac{\partial v}{\partial \nu}(y) d s(y)+\int_{\Gamma_{1}} f_{1}(y) \frac{\partial v}{\partial \nu}(y) d s(y)=0 .
$$

Since $f_{2}$ and $v$ are fixed and $f_{1}$ arbitrary, by taking $f_{1}=0$, we find that the first term in the left-hand side vanish. We are then left with

$$
\int_{\Gamma_{1}} f_{1}(y) \frac{\partial v}{\partial \nu}(y) d s(y)=0 .
$$

Since $f_{1}$ is arbitrary, we conclude that the normal derivative of $v$ vanish on $\Gamma_{1}$. From the construction, $v$ also vanish on $\Gamma_{1}$. By Holmgren's theorem, $v=0$ in $\bar{D}$, hence $z_{2}$ is zero, which is a contradiction.

To conclude, for each smooth function $f_{2}$ on $\Gamma_{2}$, there is a dense set of functions $g_{2}$ for which (8) has a solution. It therefore follows that the operator $B$ has a dense range in $L^{2}\left(\Gamma_{2}\right) \times L^{2}\left(\Gamma_{2}\right) \times\{0\}$.

We point out that the above proof of injectivity follows the steps in [1, Theorem 4.1]. Moreover, the result obtained in the denseness part stating that for each smooth function $f_{2}$ there is a dense set of functions $g_{2}$ for which (8) has a solution, is in accordance with [6, Chapt. 3, Thm. 1.1]. There it is shown that a dense set of Cauchy data for elliptic equations is obtained by taking restrictions of solutions to a mixed boundary problem.

We can then write our inverse problem as an operator equation

$$
B \phi=F
$$

to be solved for $\phi=\left(\phi_{1}, \phi_{2}, A\right)$ given the data $F=(f, g, 0)$. To restore stability, Tikhonov regularization is employed, that is we solve the regularized system

$$
\left(B^{*} B+\alpha I\right) \phi_{\alpha}=B^{*} F,
$$

where $B^{*}$ is the adjoint operator to $B$, and $\alpha>0$ is a regularization parameter to be chosen appropriately.

According to the representation (3) the Cauchy data on $\Gamma_{1}$ can be calculated as

$$
\begin{gathered}
u(x)=\frac{1}{2} \phi_{1}(x)+\left(D_{11} \phi_{1}\right)(x)+\left(D_{12} \phi_{2}\right)(x)+A G_{1}(x), \\
\frac{\partial u}{\partial \nu}(x)=\left(T_{11} \phi_{1}\right)(x)+\left(T_{12} \phi_{2}\right)(x)+A \tilde{G}_{1}(x) .
\end{gathered}
$$




\section{FUll DisCRETIZATION}

We assume that the boundary curves have the parametrization

$$
\Gamma_{i}=\left\{x_{i}(t)=\left(x_{i 1}(t), x_{i 2}(t)\right), \quad t \in[0,2 \pi]\right\},
$$

where $x_{i}: \mathbb{R} \rightarrow \mathbb{R}^{2}$ is $2 \pi$-periodic with $\left|x_{i}^{\prime}(t)\right|>0$ for all $t \in[0,2 \pi], x_{i} \in C^{3}([0,2 \pi] \times$ $[0,2 \pi]), i=1,2$.

Using these parametric representations in (6) and (7), we get the parametrized system of integral equations

$$
\left\{\begin{array}{l}
\frac{1}{2 \pi} \int_{0}^{2 \pi} \psi_{1}(\tau) K_{21}(t, \tau) d \tau-\psi_{2}(t)+ \\
\quad+\frac{1}{2 \pi} \int_{0}^{2 \pi} \psi_{2}(\tau) K_{22}(t, \tau) d \tau+2 A G_{2}\left(x_{2}(t)\right)=\tilde{f}(t) \\
\frac{1}{2 \pi} \int_{0}^{2 \pi} \psi_{1}(\tau) L_{21}(t, \tau) d \tau+\frac{1}{2 \pi\left|x_{2}^{\prime}(t)\right|}\left[\int_{0}^{2 \pi} \psi_{2}^{\prime}(\tau) \cot \frac{\tau-t}{2} d \tau+\right. \\
\left.\int_{0}^{2 \pi} \psi_{2}(\tau) L_{22}(t, \tau) d \tau\right]+A \tilde{G}_{2}\left(x_{2}(t)\right)=\tilde{g}(t) \\
\int_{0}^{2 \pi} \psi_{1}(\tau)\left|x_{1}^{\prime}(\tau)\right| d \tau=0
\end{array}\right.
$$

where $\psi_{i}(t)=\phi\left(x_{i}(t)\right), i=1,2, \tilde{f}(t)=2 f\left(x_{2}(t)\right), \tilde{g}(t)=g\left(x_{2}(t)\right)$ for $t \in[0,2 \pi]$.

Here, we used well-known relations between the normal derivative of the double-layer potential and the single-layer potential (see [11]).

Recalling the fundamental solution of the Laplace equation for planar domains, see (4), the kernels can be written as

$$
K_{i j}(t, \tau)=\frac{2\left(x_{i}(t)-x_{j}(\tau)\right) \cdot \nu\left(x_{j}(\tau)\right)}{\left|x_{i}(t)-x_{j}(\tau)\right|^{2}}\left|x_{j}^{\prime}(\tau)\right|, \quad i \neq j
$$

and

$$
\begin{array}{r}
L_{i j}(t, \tau)=\left(\frac{-2\left(x_{i}(t)-x_{j}(\tau)\right) \cdot \nu\left(x_{i}(t)\right)\left(x_{i}(t)-x_{j}(\tau)\right) \cdot \nu\left(x_{j}(\tau)\right)}{\left|x_{i}(t)-x_{j}(\tau)\right|^{4}}\right. \\
\left.+\frac{\nu\left(x_{i}(t)\right) \cdot \nu\left(x_{j}(\tau)\right)}{\left|x_{i}(t)-x_{j}(\tau)\right|^{2}}\right)\left|x_{j}^{\prime}(\tau)\right|, \quad i \neq j,
\end{array}
$$

with diagonal values

$$
K_{i i}(t, t)=\frac{x_{i}^{\prime \prime}(t) \cdot \nu\left(x_{i}(t)\right)}{\left|x_{i}^{\prime}(t)\right|}
$$

and

$$
L_{i i}(t, \tau)=\left\{\begin{array}{r}
\frac{2 x_{i}^{\prime}(t) \cdot\left(x_{i}(\tau)-x_{i}(t)\right) x_{i}^{\prime}(\tau) \cdot\left(x_{i}(\tau)-x_{i}(t)\right)}{\left|x_{i}(t)-x_{i}(\tau)\right|^{4}}-\frac{x_{i}^{\prime}(t) \cdot x_{i}^{\prime}(\tau)}{\left|x_{i}(t)-x_{i}(\tau)\right|^{2}} \\
-\frac{1}{4 \sin ^{2}\left(\frac{\tau-t}{2}\right)}, \quad t \neq \tau, \\
-\frac{1}{12}+\frac{x_{i}^{\prime}(t) \cdot x_{i}^{\prime \prime \prime}(t)}{6\left|x_{i}^{\prime}(t)\right|^{2}}+\frac{x_{i}^{\prime \prime}(t)^{2}}{4\left|x_{i}^{\prime}(t)\right|^{2}}-\frac{\left(x_{i}^{\prime}(t) \cdot x_{i}^{\prime \prime}(t)\right)^{2}}{2\left|x_{i}^{\prime}(t)\right|^{4}}, \quad t=\tau .
\end{array}\right.
$$


Chapko R., Johansson B. T., Shtoyko M.

ISSN 2078-5097. Вісн. Львів. ун-ту. Сер. прикл. матем. та інф. 2020. Вип. 28

We consider quadrature rules constructed via trigonometrical interpolation [11] with $2 n$ equidistant nodal points

$$
t_{j}=\frac{j \pi}{n}, \quad j=0, \ldots, 2 n-1,
$$

with respect to the $2 n$-dimensional space $\mathcal{T}_{n}$ of trigonometric polynomials of the form

$$
\mathcal{T}_{n}=\left\{v(s)=\sum_{m=0}^{n} a_{m} \cos m s+\sum_{m=1}^{n-1} b_{m} \sin m s, a_{m}, b_{m} \in \mathbb{R}\right\} .
$$

Denote by $\left\{\mathcal{L}_{j} \in \mathcal{T}_{n}, j=0, \ldots, 2 n-1\right\}$ the corresponding Lagrange basis.

The two quadrature rules are

$$
\frac{1}{2 \pi} \int_{0}^{2 \pi} f(\tau) \cot \frac{t-\tau}{2} d \tau \approx \sum_{k=0}^{2 n-1} \tilde{T}_{k}(t) f\left(t_{k}\right)
$$

and

$$
\frac{1}{2 \pi} \int_{0}^{2 \pi} f(\tau) d \tau \approx \frac{1}{2 n} \sum_{k=0}^{2 n-1} f\left(t_{k}\right)
$$

with the weight functions derived from [12],

$$
\tilde{T}_{j}(t)=-\frac{1}{n} \sum_{m=1}^{n-1} m \cos \left(m\left(t-t_{j}\right)\right)-\frac{1}{2} \cos \left(n\left(t-t_{j}\right)\right) .
$$

Using the Nyström method with quadratures (13) and (14) in the integral equations (11), we obtain the following system of linear equations

$$
\left\{\begin{array}{l}
\frac{1}{2 n} \sum_{j=0}^{2 n-1} \bar{\psi}_{1, j} K_{21}\left(t_{i}, t_{j}\right)+\frac{1}{2 n} \sum_{j=0}^{2 n-1} \bar{\psi}_{2, j} K_{22}\left(t_{i}, t_{j}\right)-\psi_{2, i}+2 A G_{2}\left(x_{2}\left(t_{i}\right)\right)=\bar{f}_{i} \\
\frac{1}{2 n} \sum_{j=0}^{2 n-1} \bar{\psi}_{1, j} L_{21}\left(t_{i}, t_{j}\right)+ \\
\quad+\frac{1}{\left|x_{2}^{\prime}\left(t_{i}\right)\right|} \sum_{j=0}^{2 n-1} \bar{\psi}_{2, j}\left[\tilde{T}_{j}\left(t_{i}\right)+\frac{1}{2 n} L_{22}\left(t_{i}, t_{j}\right)\right]+A \tilde{G}_{2}\left(x_{2}\left(t_{i}\right)\right)=\bar{g}_{i}, \\
\sum_{j=0}^{2 n-1} \bar{\psi}_{1, j}\left|x_{1}^{\prime}\left(t_{j}\right)\right|=0
\end{array}\right.
$$

to be solved for $\bar{\psi}_{1, j} \approx \psi_{1}\left(t_{j}\right)$ and $\bar{\psi}_{2, j} \approx \psi_{2}\left(t_{j}\right)$ with the right-hand side $\bar{f}_{i}=\tilde{f}\left(t_{i}\right)$ and $\bar{g}_{i}=\tilde{g}\left(t_{i}\right)$ for $i=0, \ldots, 2 n-1$. Rearranging (15), we arrive at the following system of linear algebraic equations:

$$
\mathbf{A x}=\mathbf{b}
$$

where the matrix $\mathbf{A} \in \mathbb{R}^{4 n+1 \times 4 n+1}$ and $\mathbf{x}=\left[\bar{\psi}_{1}, \bar{\psi}_{2}, A\right]^{\top}$ with $\mathbf{b}=[\bar{f}, \bar{g}, 0]^{\top}$. The matrix A will have a large condition number due to the ill-posedness of the Cauchy problem. To obtain a stable smooth solution regularization of this system is necessary. 
Chapko R., Johansson B. T., Shtoyko M.

As explained at the end of the previous section, to solve (16) in a stable way, we employ Tikhonov regularization; the standard version of Tikhonov regularization amounts to solve the minimization problem

$$
\min _{x}\left\{\left\|\mathbf{A} \mathbf{x}-\mathbf{b}^{\delta}\right\|_{2}^{2}+\lambda\|\mathbf{x}\|_{2}^{2}\right\}
$$

where $\lambda \in \mathbb{R}$ is a regularization parameter that has to be appropriately chosen and $\mathbf{b}^{\delta}$ are the given noisy data with error level $\delta$. The Tikhonov regularized solution $x_{\lambda}$ in (17) is equivalently given as the solution to the regularized normal equations

$$
\left(\mathbf{A}^{*} \mathbf{A}+\lambda I\right) \mathbf{x}_{\lambda}=\mathbf{A}^{*} \mathbf{b}^{\delta}
$$

where $\mathbf{A}^{*}$ is the transpose of the matrix $\mathbf{A}$. Although there are optimal choices for the regularization parameter (the discrepancy principle), it is often simpler and faster to use a heuristic choice such as the L-curve rule [8].

A convergence analysis can be carried out based on general results given for example in [10]. Denote by $\psi_{\ell, n}^{\lambda} \in \mathcal{T}_{n}, \ell=1,2$, solutions obtained via (18) as

$$
\psi_{\ell, n}^{\lambda}(t)=\sum_{j=0}^{2 n-1} \bar{\psi}_{\ell, j}^{\lambda} \mathcal{L}_{j}(t)
$$

and by $\psi_{\ell, n} \in \mathcal{T}_{n}, \ell=1,2$, solutions obtained via (16) as

$$
\psi_{\ell, n}(t)=\sum_{j=0}^{2 n-1} \bar{\psi}_{\ell, j} \mathcal{L}_{j}(t)
$$

Clearly, we have the following inequality

$$
\left\|\psi_{\ell, n}^{\lambda}-\psi_{\ell}\right\|_{L^{2}} \leq\left\|\psi_{\ell, n}^{\lambda}-\psi_{\ell, n}\right\|_{L^{2}}+\left\|\psi_{\ell, n}-\psi_{\ell}\right\|_{L^{2}}
$$

It is possible under additional assumptions on the matrix $\mathbf{A}$ to show the estimates

$$
\left\|\psi_{\ell, n}^{\lambda}-\psi_{\ell, n}\right\|_{L^{2}} \leq C \sqrt{\delta}, \quad C>0
$$

and

$$
\left\|\psi_{\ell, n}-\psi_{\ell}\right\|_{L^{2}} \leq \inf \left\{\left\|\psi_{\ell}-\xi\right\|_{L^{2}}, \xi \in \mathcal{T}_{n}\right\}
$$

Thus, in the case of exact data the error estimate of this approach corresponds to the case of well-posed hypersingular integral equations (see [12]).

The numerical value of the Cauchy data on $\Gamma_{1}$ can by calculated as

$$
u_{n}\left(x_{1}\left(t_{i}\right)\right)=\frac{1}{2} \bar{\psi}_{1, i}^{\lambda}+\frac{1}{4 n} \sum_{\ell=1}^{2} \sum_{j=0}^{2 n-1} \bar{\psi}_{\ell, j}^{\lambda} K_{1, \ell}\left(t_{i}, t_{j}\right)+A^{\lambda} G_{1}\left(x_{1}\left(t_{i}\right)\right)
$$

and

$$
\begin{array}{r}
\frac{\partial u_{n}}{\partial \nu}\left(x_{1}\left(t_{i}\right)\right)=\frac{1}{\left|x_{1}^{\prime}\left(t_{i}\right)\right|} \sum_{j=0}^{2 n-1} \bar{\psi}_{1, j}^{\lambda}\left[\tilde{T}_{j}\left(t_{i}\right)+\frac{1}{2 n} L_{11}\left(t_{i}, t_{j}\right)\right] \\
+\frac{1}{2 n} \sum_{j=0}^{2 n-1} \bar{\psi}_{2, j}^{\lambda} L_{12}\left(t_{i}, t_{j}\right) \\
+A^{\lambda} \tilde{G}_{1}\left(x_{1}\left(t_{i}\right)\right) .
\end{array}
$$


Chapko R., Johansson B. T., Shtoyko M.

\section{NUMERICAL EXPERIMENTS}

We illustrate by numerical examples the robustness, for both exact and noisy data, of the proposed double-layer method for the reconstruction of a harmonic function satisfying the Cauchy problem (1)-(2). In the case of noisy data, random pointwise errors are added to the function values $g$ on the outer boundary $\Gamma_{2}$ with the percentage given in terms of the $L^{2}$-norm. The discretization parameter controlling the number of mesh points on each boundary curve is taken as $n=32$ in (12). The value of the regularization parameter $\lambda$ is chosen by trial and error; we calculated the numerical solutions for $\lambda=10^{-m}$ with $m=1, \ldots, 15$, and use the value giving the most accurate result.

Example 1. The solution domain $D$ is bounded by the curves (see Fig. 2a)

$\Gamma_{1}=\left\{x_{1}(s)=(0.6 \cos s, 0.4 \sin s), s \in[0,2 \pi]\right\}, \quad \Gamma_{2}=\left\{x_{2}(s)=(\cos s, \sin s), s \in[0,2 \pi]\right\}$.

We consider the harmonic function $u_{e x}(x)=x_{1}^{2}-x_{2}^{2}$, with $x \in D$, as the exact solution

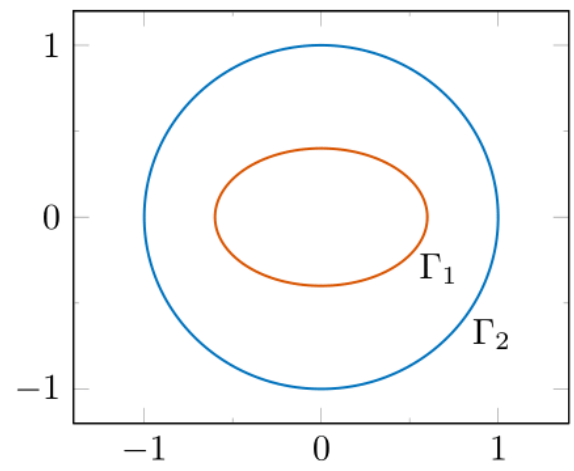

a) The domain $D$ in Ex. 1

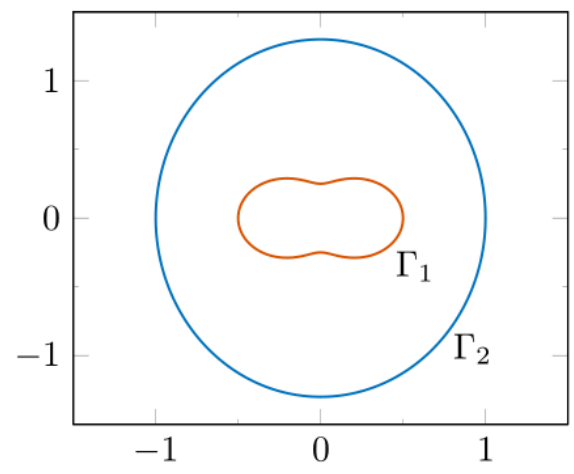

b) The domain $D$ in Ex. 2

Fig. 2. The two solution domains used in the numerical experiments

of the Cauchy problem (1)-(2). The Cauchy data is then given as

$$
f(x)=x_{1}^{2}-x_{2}^{2}, \quad g(x)=2\left(x_{1},-x_{2}\right) \cdot \nu(x), \quad x \in \Gamma_{2} .
$$

We use the discrete $L^{2}$-error $e_{2}$ as a measure of the quality of the reconstruction of the function values, where

$$
e_{2}=\left(\frac{1}{2 n} \sum_{i=0}^{2 n-1}\left[f\left(x_{1}\left(t_{i}\right)\right)-u_{n}\left(x_{2}\left(t_{i}\right)\right)\right]^{2}\right)^{1 / 2}
$$

and corresponding $L^{2}$-error $q_{2}$ to measure the quality of the reconstruction of the normal derivative.

The results of the numerical reconstruction of the Cauchy data on the interior curve $\Gamma_{1}$ with the proposed method in the case of $3 \%$ noise are presented in Figs. 3a and 3b. The solid line in each figure is the analytical value and the dashed line the corresponding approximation. The approximations will improve further with less noise and we mention that in the case of exact data, we obtain $e_{2}=2.6 E-8$ and $q_{2}=2.2 E-7$ for $\lambda=1 E-8$. 
Chapko R., Johansson B. T., Shtoyko M.

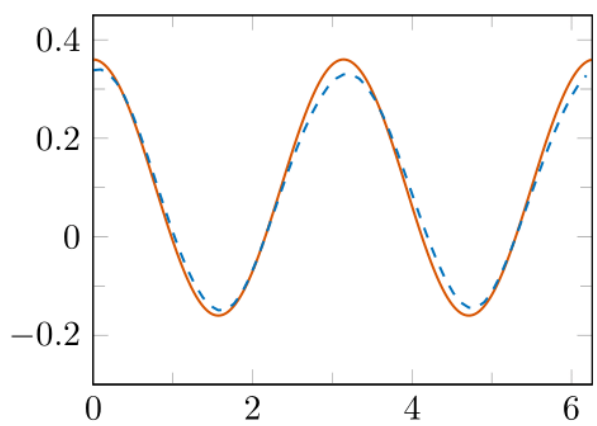

a) Function, $e_{2}=0.02$

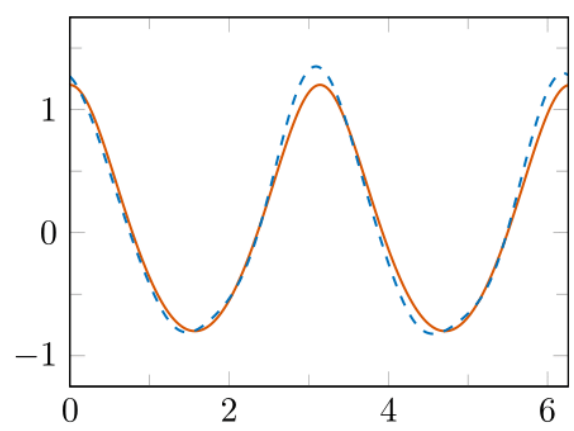

b) Normal derivative, $q_{2}=0.09$

Fig. 3. Cauchy data reconstruction on $\Gamma_{1}$ for $3 \%$ noise in the data in Ex. $1: \lambda=1 E-2$

Example 2. The domain $D$ has boundary curves given by (see Fig. 2b)

$$
\Gamma_{1}=\left\{x_{1}(s)=\left(\sqrt{(0.5 \cos s)^{2}+(0.25 \sin s)^{2}}(\cos s, \sin s), s \in[0,2 \pi]\right\}\right.
$$

and

$$
\Gamma_{2}=\left\{x_{2}(s)=(\cos s, 1.3 \sin s), s \in[0,2 \pi]\right\} .
$$

We choose as the exact solution the following harmonic function

$$
u_{e x}(x)=\Phi\left(x, y^{*}\right), \quad x \in D, \quad y^{*} \notin D
$$

with $y^{*}=(3,0)$ and $\Phi$ the fundamental solution given in (4).

In Fig. 4 are the results of the reconstruction of the Cauchy data on the boundary $\Gamma_{1}$ in the case of $3 \%$ noise. For exact data, we obtained $e_{2}=1.7 E-7$ and $q_{2}=8.1 E-6$ for $\lambda=1 E-10$.

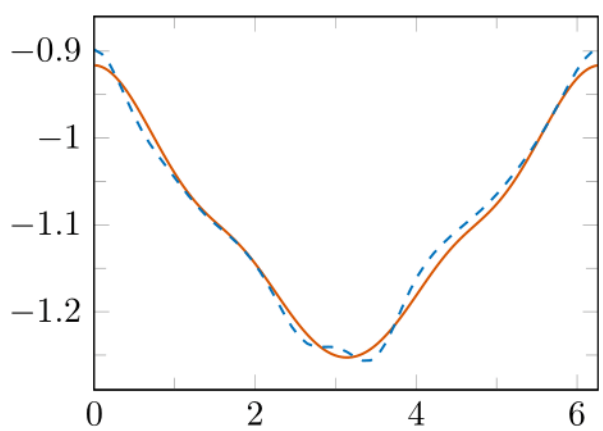

a) Function, $e_{2}=0.01$

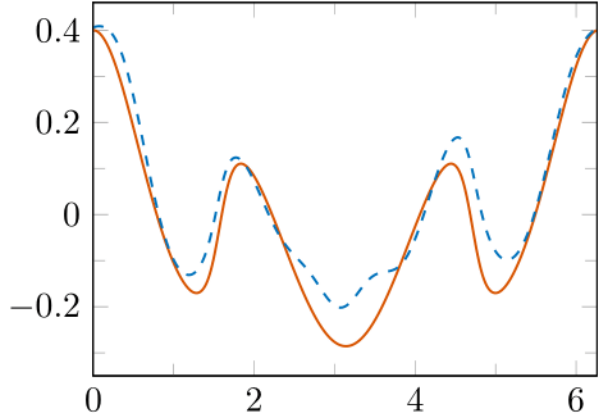

b) Normal derivative, $q_{2}=0.07$

Fig. 4. Cauchy data reconstruction on $\Gamma_{1}$ for $3 \%$ noise in the data in Ex.2: $\lambda=1 E-3$

As we see in Fig 3 and Fig 4 the reconstruction of the function values on $\Gamma_{1}$ has high accuracy also for noisy data. It is pleasing to see that also the normal derivative is reconstructed with acceptable accuracy. Differentiation is itself an ill-posed problem, thus finding numerically the normal derivative is an additional challenge compared to finding the function values. 
Chapko R., Johansson B. T., Shtoyko M.

\section{Conclusions}

The numerical solution of the planar Cauchy problem for the Laplace equation in doubly connected domains is considered. Using a double-layer potential approach the Cauchy problem is reduced to a system of ill-posed integral equations having a hypersingularity in the kernels. The injectivity of the corresponding linear operator together with denseness of its range are shown. The full discretization is realized by a trigonometrical Nyström type method. It leads to a linear system which is solved by Tikhonov regularization. The numerical experiments confirmed the usability of proposed approach including in the case of noisy data for generating a stable solution to the Cauchy problem.

\section{REFERENCES}

1. Cakoni $F$. Integral equations for inverse problems in corrosion detection from partial Cauchy data, / F. Cakoni, R. Kress // Inverse Probl. Imaging. - 2007. - Vol. 1. - P. 229-245.

2. Chapko R. On the numerical solution of a Cauchy problem for the Laplace equation via a direct integral equation approach / R. Chapko, B. T. Johansson // Inverse Probl. Imaging.2012. - Vol. 6. - P. 25-38.

3. Chapko R. A boundary integral approach for numerical solution of the Cauchy problem for the Laplace equation / R. Chapko, B. T. Johansson // Ukraïn. Mat. Zh.- 2016.- Vol.68.P. 1665-1682.

4. Chapko R. A boundary integral equation method for numerical solution of parabolic and hyperbolic Cauchy problems / R. Chapko, B. T. Johansson // Appl. Numer. Math.- 2018.Vol. 129. - P. 104-119.

5. Chapko $R$. Wave propagation from lateral Cauchy data using a boundary element method / R. Chapko, B. T. Johansson, Y. Muzychuk, A. Hlova // Wave Motion.- 2019.- Vol.91.doi.org/10.1016/j.wavemoti.2019.102385.

6. FursikovA. V. Optimal Control of Distributed Systems: Theory and Applications / A. V. Fursikov // AMS. Providence. RI. - 2000. - (Translated from the 1999 Russian original).

7. Greenbaum A. Laplace's equation and the Dirichlet-Neumann map in multiply connected domains / A. Greenbaum, L. Greengard, G. B. McFadden, // J. Comput. Phys.- 1993.Vol. 105. - P. 267-278.

8. Hansen P. C. The $L$-curve and its use in the numerical treatment of inverse problems / P. C. Hansen // In: Ed. P. Johnston Computational Inverse Problems in Electrocardiology. - Southampton: WIT Press, 2000.- P. 119-142.

9. Helsing J. Laplace's equation and the Dirichlet-Neumann map: A new mode for Mikhlin's method, / J. Helsing, E. Wadbro, // J. Comput. Phys. - 2005. - Vol. 202. - P. 391-410.

10. Kirsch A. An Introduction to the Mathematical Theory of Inverse Problems. Second edition / A. Kirsch. - New York: Springer-Verlag, 2011.

11. Kress R. Linear Integral Equations. Third Edition / R. Kress Heidelberg: Springer-Verlag, 2013.

12. Kress $R$. On the numerical solution of a hypersingular integral equation in scattering theory, / R. Kress // J. Comput. Appl. Math.- 1995.- Vol.61.- P. 345-360.

13. Mikhlin S. G. Integral Equations and their Applications to certain Problems in Mechanics, Mathematical Physics and Technology. 2nd ed. / S. G. Mikhlin.- New-York: Pergamon Press, 1964. 
14. Sun $Y$. Indirect Boundary Integral Equation Method for the Cauchy Problem of the Laplace Equation / Y. Sun // J. Sci. Comput. - 2017. - Vol. 71. - P. 469-498.

Article: received 23.09.2020

revised 15.10 .2020

printing adoption 19.10 .2020

\title{
МЕТОД ПОТЕНЦАЛУ ПОДВІЙНОГО ШАРУ ДЛЯ ПЛОСКИХ ЗАДАЧ КОШІ ДЛЯ РІВНЯННЯ ЛАПЛАСА
}

\author{
Р. Хапко ${ }^{1}$, Б. Т. Йогансон ${ }^{2}$, М. Штойко ${ }^{1}$ \\ ${ }^{1}$ Львівсъкий націоналъний університет імені Івана Франка, \\ вул. Університетсъка, 1, Лъвів, 79000, e-mail: roman.chapko@lnu.edu.ua \\ ${ }^{2}$ Математика, ІТН Кампус Норрчепіне, \\ Університет Лінчепінг, 601 74, Норрчепінг, Швеція, \\ e-mail: tomas.johansson@liu.se
}

Розглядається крайова задача Коші для рівняння Лапласа в плоскій двозв'язній області. Мета - реконструювати дані Коші на внутрішній межі за відомими даними Коші на зовнішній межі. Це приклад лінійної некоректної оберненої задачі. Використовуючи непрямий метод інтегральних рівнянь, який грунтується на поданні розв'язку задачі Коші у формі потенціалу подвійного шару, отримано систему інтегральних рівнянь щодо двох невідомих густин. З'ясовано, що ця система має єдиний розв'язок для щільної множини даних. Далі виконано параметризацію системи до періодичних інтегральних рівнянь і виділено наявну гіперсобливість у вигляді спеціальної вагової функції. Чисельне розв'язування інтегральних рівнянь реалізовано методом Нистрьома 3 тригонометричними квадратурами. У підсумку отримано повністю дискретну систему лінійних рівнянь стосовно апроксимаційних значень невідомих густин у квадратурних вузлах. Позаяк ця система некоректна, то для ї̈ розв'язування застосовано метод регуляризації Тіхонова. Параметр регуляризації вибрано шляхом перебору. Це дало змогу отримати стійкий розв'язок. Вплив дискретизації з'ясовано в короткому аналізі похибки. Результати чисельних експериментів реконструкції функції та її нормальної похідної на внутрішній межі засвідчують, що стійкі наближення можна отримати методом потенціалу подвійного шару і у випадку даних Коші з шумом. у випадку даних з шумом випадкова похибка додавалась до значень функції на зовнішній межі у відсотках, нормованих у середньо-квадратичній нормі.

Ключові слова: задача Коші, потенціал подвійного шару, гіперсингулярне інтегральне рівняння, метод Нистрьома, регуляризація Тіхонова. 